\title{
Soil Suitability Evaluation for Tobacco Based on Grey Cluster Analysis
}

\author{
Gao Rui ${ }^{1}$, Qiao Hong-bo ${ }^{1}$, Zhang Hui ${ }^{1}$, Su Yong-shi ${ }^{2}$, and Chen-Yanchun ${ }^{2}$ \\ ${ }^{1}$ College of Information and Management, Henan Agricultural University, \\ Zhengzhou 450002, China \\ ${ }^{2}$ Sanmenxia Branch of Henan Tobacco Company, Sanmenxia, 472000, China
}

\begin{abstract}
Suitability evaluation of soil for tobacco is the base of spatial analysis and optimization disposition. It provides scientific basis for reasonable development of soil for tobacco. Taking soil in San Menxia city of Henan province as a study of object, five factors which had relation of tobacco growth are adopted, grey cluster method are carried out to appraise suitability evaluation. The result shows that some soil area of Lingbao, Mianchixian and Shanxian are higher suitability areas that contain higher organic matter and $\mathrm{K}$ contents , while the soil area of Lushixian has lower organic matter and $\mathrm{N}$ contents are less suitability area. Compared with traditional methods, grey cluster method determined the weight based on factors. The result is objective reasonably.
\end{abstract}

Keywords: soil nutrients; suitability evaluation; grey cluster method.

\section{Introduction}

Soil planting tobacco suitability evaluation aims to optimally allocate crop planting through measuring the coupling of designating crop to given tobacco land, and considers simultaneously soil physical features and current/future land use patterns (Qiu, et al., 2005) .Soil condition is the base of top quality tobacco engineering and the main environmental factor that affects the quality of tobacco leaves(Zhang, et al.,2003).Soil suitability evaluation for tobacco has important practical significance that not only fully and rationally use natural resource and further develop land potential, but also promote social economic sustainable development. Qiu researched dynamic soil suitability evaluation by using artificial neural network method and discussed how to realize soil suitability evaluation in time and space (Qiu, et al., 2002); Liu studied the implementation method of expert system for soil suitability evaluation (Liu, et al., 2001); Chen studied the soil fertility suitability evaluation for tobacco in Henan province by using the methods of AHP and fuzzy mathematics (Chen, et al.,2007); Wang analyzed spatial variability characteristics of tobacco-planting soils and made suitability evaluation (Wang, et al.,2008).Soil suitability evaluation used the methods of parametric, geo-statistics(Chen, et al.,2003), fuzzy mathematics(Chen, et al.,2007) had been carried out in china. But the evaluation with the method of grey cluster is rarely reported. 
The tobacco-growing distribution area is from 300 to $1500 \mathrm{~m}$ above sea level, topography complex and soil nutrient is of great spatial variability. This study take Sanmenxia soil as study object use grey cluster method to evaluate soil nutrient suitability to detect soil nutrient. The result will provide reference for rational distribution of tobacco, balanced fertilization and tobacco sustainable development.

\section{Material and Method}

\subsection{Study Area}

Sanmenxia is the leading tobacco-producing areas of quality, is located in inland midlatitude areas, a warm temperate continental monsoon climate. The annual average temperature $13.2{ }^{\circ} \mathrm{C}$, annual average 2354.3 hours of sunshine, frost-free period of $184 \sim 218$ days, with an average annual rainfall $550 \sim 800 \mathrm{~mm}$, is suitable for the growth of flue-cured tobacco district.

\subsection{Sample Collection}

The method in collecting soil samples was used according to the second national soil survey. Sanmenxia in the choice of representative tobacco soil, topsoil from $0 \sim 20 \mathrm{~cm}$ soil samples, each sampling point of the area on behalf of $20 \mathrm{hm} 2$. In 2007 collecting soil samples 299 were made.

\subsection{Study Method}

Grey fixed weight cluster method is the method that according to whitenization weight function of index gathering a number of observed objects into several defined types, it empowers the cluster index prior. Grey fixed weight cluster can be carried out based on the following steps (Liu, et al., 2004):

Firstly, according to available indicators of observation, we define k subclass whitenization weight function of $\mathrm{j}$ index $f_{j}^{k}(\bullet)(j=1,2, \wedge, m ; k=1,2, \wedge, s)$.

Secondly, we determine cluster weight of every index $\eta_{j}(j=1,2, \wedge, m)$.

Thirdly, according to the whitenization weight function $f_{j}^{k}(\bullet)(j=1,2, \wedge, m$; $k=1,2, \wedge, s)$, cluster weight $\eta_{j}(j=1,2, \wedge, m)$ derived from the previous two steps ,as well as observation $x_{i j}(i=1,2, \wedge, n ; j=1,2, \wedge, m)$ of object $\mathrm{i}$ on $\mathrm{j}$ index, we can calculate the coefficient of grey fixed weight $\sigma_{j}^{k}=\sum_{j=1}^{m} f_{j}^{k}\left(x_{i j}\right) \bullet \eta_{j}, \quad i=1,2, \wedge, n ; k=1,2$, $\bigwedge, s$.

Finally, if $\max _{1 \leq k \leq s}\left\{\sigma_{i}^{k}\right\}=\sigma_{i}^{k^{*}}$ you can conclude that object i belong to grey category $k^{*}$. 


\section{Soil Suitability Evaluation}

\subsection{Selection of Evaluation Index}

According to checking related literature and listening to experience and views of experts, based on the principles of influence that made tobacco quality and growth, variance degree, stability and operability, we finally select 5 indexes: $\mathrm{PH}$, organic, available $\mathrm{N}$, available $\mathrm{P}$ and available $\mathrm{K}$.

\subsection{Delineation of Suitability Standard}

According to the evaluation of soil nutrient standards (Ren, et al., 2007, Li, et al.,2008) and the actual situation of tobacco-growing areas in Sanmenxia, each index will be divided into three levels: most appropriate, suitable and unsuitable corresponding to 1 3(Table 1).

Because soil $\mathrm{PH}$ value changes between 6.6 8.8 in tobacco-growing areas of Sanmenxia, the data should be standardized to positively correlate with evaluation standard

Table 1. Soil nutrient suitability evaluation standard

\begin{tabular}{cccc}
\hline index & unsuitable & suitable & most appropriate \\
\hline organic $\left(\mathrm{g} \cdot \mathrm{kg}^{-1}\right)$ & $<5$ & $5-10$ & $>10$ \\
available $\mathrm{N}\left(\mathrm{mg} \cdot \mathrm{kg}^{-1}\right)$ & $<25$ & $25-40$ & $>40$ \\
available $\mathrm{P}(\mathrm{mg} \cdot \mathrm{kg}-1)$ & $<10$ & $10-20$ & $>20$ \\
available $\mathrm{K}\left(\mathrm{mg} \cdot \mathrm{kg}^{-1}\right)$ & $<80$ & $80-150$ & $>150$ \\
$\mathrm{PH}$ & $<4.5$ & $4.5-5.5$ & $5.5-8.0$ \\
\hline
\end{tabular}

\subsection{Identification of Whitenization Weight Function}

We will divide five evaluation index into three gray class: most appropriate, suitable, unsuitable in accordance with their respective evaluation criteria, then $\mathrm{j}(\mathrm{j}=1,2,3,4,5)$ index $\mathrm{k}(\mathrm{k}=1,2,3)$ gray class indicate the $\mathrm{j}$ index the $\mathrm{k}$ gray class. Then, we set whitenization weight function of $\mathrm{j}$ index $\mathrm{k}$ gray class:

$$
f_{j}^{k}(\bullet)(j=1,2,3,4,5 ; k=1,2,3)
$$

Commonly used whitenization weight functions are typical, upper limit measure, moderate measure, lower limit measure and triangle whitenization weight function in grey system theory. Here, we select upper limit measure whitenization weight function measure the most grey class, moderate measure whitenization weight function measure the suitable grey class, lower limit measure whitenization weight function measure the unsuitable grey class. Based on the soil conditions, we determine the whitenization weight function as follows: 


$$
\begin{aligned}
& f_{1}^{1}[0,20,-,-], f_{1}^{2}[0,13,-, 20], f_{1}^{3}[-,-, 11,13] \\
& f_{2}^{1}[0,65,-,-], f_{2}^{2}[0,50,-, 65], f_{2}^{3}[-,-, 45,50] \\
& f_{3}^{1}[0,20,-,-], f_{3}^{2}[0,14,-, 20], f_{3}^{3}[-,-, 10,14] \\
& f_{4}^{1}[0,250,-,-], f_{4}^{2}[0,180,-, 250], f_{4}^{3}[-,-, 120,180] \\
& f_{5}^{1}[0,2.2,-,-], f_{5}^{2}[0,2.0,-, 2.2], f_{5}^{3}[-,-, 1.6,2.0]
\end{aligned}
$$

Based on the above whitenization weight function we can write specific function expression. Here only given whitenization weight function expression of soil organic index three grey classes and other expression can be written similarly.

$$
\begin{aligned}
f_{1}^{1}(x) & = \begin{cases}0, & x<0 \\
\frac{x}{20}, & x \in[0,20] \\
1, & x>20\end{cases} \\
f_{1}^{2}(x) & =\left\{\begin{array}{cc}
0, & x \notin[0,20] \\
\frac{x}{13}, & x \in[0,13] \\
\frac{20-x}{20-13}, & x \in[13,20]
\end{array}\right. \\
f_{1}^{3}(x) & =\left\{\begin{array}{cc}
0, & x \notin[0,13] \\
1, & x \in[0,11] \\
\frac{13-x}{2}, & x \in[11,13]
\end{array}\right.
\end{aligned}
$$

\subsection{Cluster Results and Soil Suitability Evaluation}

Based on the survey data of tobacco fields , combined with observations and recommendations of relevant experts, the weight of organic, available $\mathrm{N}$, available $\mathrm{P}$, available K, PH value are: $\eta_{1}=0.45, \eta_{2}=0.15, \eta_{3}=0.08, \eta_{4}=0.150, \eta_{5}=0.17$.

Whitenization weight function $f_{j}^{k}(\bullet)(j=1,2, \wedge, m ; k=1,2, \wedge, s)$, cluster weight $\eta \mathrm{i}(j=1,2, \wedge, m)$ and observation $x_{i j}(i=1,2, \wedge, n ; j=1,2, \bigwedge, m)$ of object $\mathrm{i}$ for $\mathrm{j}$ index calculate the grey fixed cluster coefficient. $\sigma_{i}^{k}=\sum_{j=1}^{m} f_{j}^{k}\left(x_{i j}\right) \bullet \eta_{j}, i=1,2, \wedge, n ; k=1,2, \wedge, s$.

The cluster coefficient of three types can be made according to sample data and the above steps as shown in table 2.As the sample points located in every township, the township as a unit were to be analyzed. From the result, the areas that have the most 
Table 2. Soil suitability evaluation cluster coefficient in Sanmenxia area

\begin{tabular}{|c|c|c|c|c|}
\hline $\begin{array}{c}\text { serial } \\
\text { number }\end{array}$ & township & $\begin{array}{l}\text { most suitable cluster } \\
\text { coefficient }\end{array}$ & $\begin{array}{l}\text { suitable cluster } \\
\text { coefficient }\end{array}$ & $\begin{array}{l}\text { unsuitable cluster } \\
\text { coefficient }\end{array}$ \\
\hline 1 & guandaokouzhen & 0.69 & 0.91 & 0.10 \\
\hline 2 & duguanzhen & 0.81 & 0.58 & 0.17 \\
\hline 3 & hengjianxiang & 0.69 & 0.78 & 0.65 \\
\hline 4 & fanlizhen & 0.69 & 0.78 & 0.27 \\
\hline 5 & wenyuxiang & 0.67 & 0.78 & 0.70 \\
\hline 6 & dongmingzhen & 0.75 & 0.72 & 0.26 \\
\hline 7 & shahexiang & 0.65 & 0.78 & 0.32 \\
\hline 8 & panhexiang & 0.69 & 0.95 & 0.24 \\
\hline 9 & mutongxiang & 0.87 & 0.37 & 0.06 \\
\hline 10 & mokouxiang & 0.72 & 0.84 & 0.17 \\
\hline 11 & xujiawan & 0.86 & 0.54 & 0.00 \\
\hline 12 & shuanghuaishu & 0.75 & 0.64 & 0.15 \\
\hline 13 & shizipingxiang & 0.85 & 0.24 & 0.23 \\
\hline 14 & guanpozhen & 0.92 & 0.29 & 0.00 \\
\hline 15 & wayaogouxiang & 0.68 & 0.73 & 0.23 \\
\hline 16 & tanghexiang & 0.57 & 0.79 & 0.87 \\
\hline 17 & wulichuanzhen & 0.83 & 0.53 & 0.17 \\
\hline 18 & zhuyangguanzhen & 0.84 & 0.57 & 0.07 \\
\hline 19 & caiyuanxiang & 0.72 & 0.92 & 0.01 \\
\hline 20 & dayanwaxiang & 0.64 & 0.87 & 0.30 \\
\hline 21 & gongqianxiang & 0.70 & 0.69 & 0.11 \\
\hline 22 & xilicun & 0.63 & 0.76 & 0.62 \\
\hline 23 & chencunxiang & 0.69 & 0.84 & 0.35 \\
\hline 24 & guoyuanxiang & 0.77 & 0.67 & 0.13 \\
\hline 25 & hongyangzhen & 0.81 & 0.45 & 0.14 \\
\hline 26 & potouxiang & 0.68 & 0.83 & 0.35 \\
\hline 27 & rencunxiang & 0.81 & 0.46 & 0.19 \\
\hline 28 & tianchizhen & 0.61 & 0.74 & 0.21 \\
\hline 29 & xiyangxiang & 0.66 & 0.86 & 0.42 \\
\hline 30 & yangshaoxiang & 0.76 & 0.87 & 0.24 \\
\hline 31 & yinghaozhen & 0.66 & 0.71 & 0.31 \\
\hline 32 & chuankouxiang & 0.76 & 0.69 & 0.08 \\
\hline 33 & suncunxiang & 0.74 & 0.79 & 0.28 \\
\hline 34 & wumiaoxiang & 0.86 & 0.45 & 0.00 \\
\hline 35 & zhuyangzhen & 0.87 & 0.37 & 0.00 \\
\hline
\end{tabular}


suitability are: Duguanzhen, Dongmingzhen, Mutongxiang, Xujiawan, Shuanghuaishu, Shizipingxiang, Guanpozhen, Wulichuanzhen, Zhuyangguanzhen of Lushixian; Gongqianxiang of Shanxian; Guoyuanxiang, Hongyangzhen, Rencunxiang of Mianchixian; Wumiaoxiang, Zhuyangzhen of Lingbao. The Soil of these 15 townships organic and soil $\mathrm{K}$ content is high, soil $\mathrm{pH}$ and $\mathrm{N}$ content is medium. The number of sample point account for $29.4 \%$ in total.

Suitable soil areas are: Guandaokouzhen, Hengjianxiang, Fanlizhen, Wenyuxiang, Shahexiang, Mokouxiang, Wayaogouxiang of Lushixian; Caiyuanxiang, Dayanwaxiang, Xilicunxiang of Shanxian; Chencunxiang, Potouxiang, Tianchizhen, Xiyangxiang, Yangshaoxiang, Yinghaozhen of Mianchixian; Chuankouxiang, Sucunxiang of Lingbao. Although soil K content is high in these areas, soil organic content is lower in some parts, soil improvement should be pay attention to in the future. The point number is $210(70.4 \%)$.

Tanghexiang locate in Lushixian has poor soil suitability. The soil organic content is lower, $\mathrm{pH}$ value is higher, $\mathrm{N}$ and $\mathrm{P}$ content is lower.

Generally, soil planting tobacco suitability is good in Sanmenxia that suitable for the growth of high-quality tobacco leaf. In recent years, soil in this area $\mathrm{pH}$ value, $\mathrm{N}$ content has an upward trend, pay attention to balanced fertilization to ensure harmonious proportion of $\mathrm{N}, \mathrm{P}, \mathrm{K}$ content.

\section{Conclusions}

Gray clustering method for soil suitability evaluation is a new attempt, by choosing its evaluation index, determining whitenization weight function to carry out soil nutrient gray clustering. In this paper, using above method, the study of Sanmenxia tobacco soil suitability assessment carried out. The results show that almost $30 \%$ area of this region has better soil suitability. Less than $1 \%$ has poor foil suitability, rest area has medium suitability. Taking into account the soil itself is a complex multi-phase organic-inorganic complex, involving the interaction of various factors, the evaluation results must be combining with the actual production, index weight and the determination of whitenization weight function is still value the experience of using the traditional method. The evaluation results basically tallies with actual production. Soil $\mathrm{pH}$ value and available $\mathrm{N}$ content are higher in Sanmenxia, Attention should be given to improve soil, reduce soil $\mathrm{pH}$ value, $\mathrm{CL}$ content, control $\mathrm{N}$ content, complement $\mathrm{P}$ content and stabilize $\mathrm{K}$ content.

\section{References}

[1] Qiu, B.W., et al.: Fruit tree suitability assessment using GIS and multi-criteria evaluation. Transaction of the CSAE 21(6), 667-690 (2005)

[2] Zhang, Y., et al.: Oriental tobacco from different region and the intrinsic chemical composition analysis of aroma substances. Chinese Tobacco Science (4), 12-16 (2003)

[3] Qiu, B.W., et al.: Dynamic assessment of regional land resource suitability based on geographical information system. Journal of Soil 39(3), 301-307 (2002)

[4] Liu, Y.Z., et al.: Establishment of GIS-supported expert system for soil suitability evaluation. Chinese Journal of Soil Science 32(5), 193-196 (2001) 
[5] Chen, H.S., et al.: Comprehensive evaluation of soil fertility suitability for tobacco based on GIS in Nanyang city, He'nan province. System Science and Comprehensive Studies in Agriculture 23(4), 498-502 (2007)

[6] Chen, H.S., et al.: Comprehensive evaluation system of soil fertility suitability in Xuchang tobacco planting regions based on GIS. Journal of Henan University (Natural Science) 39(1), 51-56 (2009)

[7] Wang, Z.F., et al.: Spatial variability of soil nutrient and its suitability evaluation in tobacco planted area. Journal of Southwest University (Natural Science Edition) 30(1), 98 102 (2008)

[8] Chen, W.H., et al.: Spatial variability analysis for environmental information of fields. Eco-Journal 24(2), 347-351 (2004)

[9] Chen, H.S., et al.: Comprehensive fertility evaluation of soil for tobacco plantation in Henan province based on GIS. Chinese Journal of Soil Science 38(6), 1081-1085 (2007)

[10] Liu, S.F., et al.: Gray system theory and its application. Science Press, Beijing (2004)

[11] Ren, Z., et al.: Practical method of appraising soil suitability of planting tobacco in Guizhou. Journal of Anhui Agriculture Science 35(31), 9955-9956 (2007)

[12] Li, X.H., et al.: Feasibility of climate and soil in Enshi tobacco growing areas. Chinese Tobacco Science 29(5), 18-21 (2008) 